Methods 31 babies born between 28 and 36 weeks' gestation were studied. 19 babies were IUGR with individualised birth weight (normalised for sex, ethnicity, parity, maternal BMI) below $3^{\text {rd }}$ centile. 12 babies were appropriately grown with individualised birth weight between the $25^{\text {th }}$ and $75^{\text {th }}$ centile. Blood neurotrophin concentrations were measured using protein chip technology in 30 babies (19 IUGR and 11 controls) between 2 and 7 days after birth. In 14 babies ( 7 from each group) MRI brain was performed at term equivalence.

Results Fractional anisotropy (FA) was lower in IUGR babies compared to controls in 7 out of 8 regions with no statistical significance. Apparent diffusion coefficient (ADC) was lower in IUGR babies compared to controls in 6 out of 8 regions, reaching significance in frontal lobes. 7 out of 8 regions were smaller in IUGR babies compared to the control babies, reaching significance in the dorso-medial pre-frontal cortex. Differences did not persist when normalised for intracranial volume. Serum neurotrophin concentrations were elevated in IUGR babies but did not reach statistical significance. Using multiple regression only FA of right frontal lobe was significantly related to $B D N F(R=0.65$; $\mathrm{p}=0.012$ )

Conclusion IUGR babies showed no differences in neurotrophin concentrations and decreased $\mathrm{ADC}$ in frontal lobes when compared with controls.

\section{PREDICTIVE VALUE OF VASCULAR ENDOTHELIAL GROWTH FACTOR IN PRETERM NEONATES WITH INTRAVENTRICULAR HAEMORRHAGE}

doi:10.1136/archdischild-2012-302724.1091

S Atef, S Hassanin, M El Chimi, M Mohamed. Ain Shams University, Cairo, Egypt

Objective Intraventricular haemorrhage (IVH) is a major problem in premature infants. Our objective is to assess the early predictive value of vascular endothelial growth factor (VEGF) for development of IVH and management of its squeal in preterm neonates.

Methods We prospectively studied 150 preterm neonates (PT) less than 34 weeks gestation. Fifty of them completed the study. 30/50 developed IVH during follow up, and 20 did not. First 24 hours, and 3rd day serum samples were collected. Cerebrospinal fluid (CSF) samples were withdrawn for $10 \mathrm{IVH}$ patients.

Results Serum VEGF; both samples were increased in IVH compared to non-IVH group, $(\mathrm{P}=0.001)$. PHVD-group $(\mathrm{n}=10)$ had higher VEGF in both samples than resolved IVH $(\mathrm{P}=0.004),(\mathrm{P}=0.005)$. While, VEGF increased in the IVH group 2nd sample compared to 1 st $(\mathrm{P}=0.000)$, it decreased in non-IVH group, $\mathrm{P}=0.033)$. Each 1 unit increase in 1ST VEGF increased the risk of occurrence of IVH by $1.6 \%$. 3rd day VEGF at a cut-off value of $135 \mathrm{pg} / \mathrm{ml}$ is $96 \%$ sensitive and 100 specific to predict post haemorrhagic ventricular dilatation (PHVD). Serum VEGF inversely correlated with TLC, pH, PO2 and $\mathrm{HCO} 3$, and positively correlated with $\mathrm{PCo} 2$ and $\mathrm{FiO} 2$.

Conclusion Serum VEGF predicts development of IVH and PHVD in PT neonates. Also, high CSF level of VEGF could predict the need for permanent shunt placement.

\section{NEONATAL HYPOGLYCEMIA IS ASSOCIATED WITH INCREASED ALBUMIN NITRATION IN SMALL FOR GESTATIONAL AGE TERM NEWBORNS}

doi:10.1136/archdischild-2012-302724.1092

'N Lefevre, ' $\mathrm{JL}$ Wayenberg, ${ }^{2} \mathrm{C}$ Ghaddhab, ${ }^{2} \mathrm{D}$ Vermeylen, ${ }^{3} \mathrm{E}$ Damis, ${ }^{3} \mathrm{M}$ Flausch, ${ }^{4} \mathrm{~S}$ Bottari. 'Department of Pediatrics, Hôpital Universitaire des Enfants Reine Fabiola; ${ }^{2}$ Neonatal Intensive and Non-Intensive Care Unit, Hôpital Erasme; ${ }^{3}$ Neonatal Intensive and Non-Intensive Care Unit, CHIREC, Brussels, Belgium; ${ }^{4}$ Department of Biology and Pathology, CHU \& Laboratoire de Bioénergétique Fondamentale et Appliquée - INSERM U1055, Université Joseph Fourier, Grenoble, France
Background and Aims Neonatal hypoglycemia is a frequent event in small for gestational age (SGA) term newborns. Its clinical significance is a highly controversial issue but in experimental models, hypoglycemia has been reported to cause oxidative stress. Among the reactive species, peroxynitrite is responsible for protein nitration, lipid peroxydation and DNA damage, a process referred to as nitro-oxidative stress which can induce apoptosis. The aim of the present study was to investigate whether hypoglycemia is associated with plasma albumin nitration as a marker of nitro-oxidative stress in SGA term newborns.

Methods Using a highly sensitive ELISA we quantified plasma nitroalbumin (PNA) as a marker of peroxynitrite generation in 26 SGA term newborns with close glucose monitoring. We compared PNA concentrations in 9 normoglycemic (glycemia $\geq 2.5 \mathrm{mmol} / \mathrm{L}$ ) newborns and in 17 hypoglycemic (glycemia $<2.5 \mathrm{mmol} / \mathrm{L}$ ) newborns.

Results PNA measured during the first hours of life was inversely correlated with glycemia $(r=-0.63, p=0.01)$ and significantly higher in hypoglycemic compared to normoglycemic patients $(7.4 \mathrm{ng} / \mathrm{mL}$ [5.0-7.9] in normoglycemic patients vs. $20.8 \mathrm{ng} / \mathrm{mL}$ [9.9-77.7] in hypoglycemic patients, $\mathrm{n}=14, \mathrm{p}<0.01)$. PNA measured at day 1 of life was significantly higher in patients with recurrent hypoglycemia compared to patients with transient hypoglycemia and normoglycemic patients. We observed significant correlations between PNA at day 1 and the area under the curve of the glycemia measured before PNA sampling for several threshold values of glycemia.

Conclusions These results indicate that recurrent hypoglycemia is associated with systemic protein nitration in SGA term newborns, suggestive of a significant nitro-oxidative stress.

\section{HYPOGLYCEMIA INDUCES A NITRO-OXIDATIVE STRESS IN} PRETERM NEWBORNS

doi:10.1136/archdischild-2012-302724.1093

${ }^{1} \mathrm{C}$ Ghaddhab, ${ }^{1} \mathrm{~N}$ Lefèvre, ${ }^{1} \mathrm{JL}$ Wayenberg, ${ }^{2,3} \mathrm{SP}$ Bottari. 'Department of Pediatrics, Université Libre de Bruxelles, Brussels, Belgium; ${ }^{2}$ Fundamental and Applied Bioenergetics (LBFA), INSERM U1055, and Université Joseph Fourrier, Grenoble, St Martin d'Heres; ${ }^{3}$ Medicinal Chemistry, CHU Grenoble, Grenoble, France

Recent data suggest that free radical injury occurs in the neonatal brain after hypoglycemia in animal models. We developed an ELISA allowing the quantitative determination of nitrated plasma albumin (nitroalbumin, PNA) as a biomarker of peroxynitrite generation and investigated the potential nitro-oxidative stress induced by hypoglycemia $(<2.5 \mathrm{mmol} / \mathrm{L})$ in premature newborns.

We measured PNA concentrations at days 0,1 and 4 of life in 72 preterm infants without any other obvious cause of nitro-oxidative stress such as infection, asphyxia and hyperglycemia. Glucose levels were monitored every 3-4 hours using a strip method. For each patient, we calculated the AUCs for glycaemia measured during the 12,18 and 24 hours preceding blood sampling as an index of the severity, the number and the duration of hypoglycemic events during the first day of life. Statistical analysis was performed using non-parametric tests.

PNA concentrations were significantly higher in hypoglycaemic than in normoglycemic infants at days 0,1 and 4. A significant inverse correlation was found between PNA at $\mathrm{D}_{1}$ and AUGs. PNA concentration at $D_{1}$ is related to the number of hypoglycemic events. Gender, term, oxygen exposure, respiratory and hemodynamic parameters were not correlated with PNA concentrations.

Thus, low glycemia levels during the first day of life are specifically associated with increased albumin nitration in preterm newborns, especially in case of recurrent hypoglycemia. This suggests the occurrence of systemic nitro-oxidative stress implying a risk of end-organ damage due to protein nitration, lipid peroxidation and DNA damage, in particular to the brain. 
1094

\section{CORD BLOOD BRAIN DERIVED NEUROTROPHIC FACTOR: DIAGNOSTIC AND PROGNOSTIC MARKER IN FULLTERM NEWBORNS WITH PERINATAL ASPHYXIA}

doi:10.1136/archdischild-2012-302724.1094

S Atef, G Gad, S Imam, M Shawky. Ain Shams University, Cairo, Egypt

Backgrounds This prospective case control study was designed to evaluate cord blood brain derived neurotrophic factor level in full term newborns with perinatal asphyxia as a marker of central nervous system insult and predictor of severity of hypoxic ischemic encephalopathy, with follow up of its level during the reperfusion phase.

Material and Methods The study included twenty fullterm neonates with perinatal asphyxia (cases) and twenty controls. Cord blood samples were obtained at birth and peripheral blood samples at $72 \mathrm{~h}$ postnatal from cases only. Plasma brain derived neurotrophic factor level was measured using enzyme linked immunosorbent assay. The clinical severity of encephalopathy was graded based on Sarnat and Sarnat staging.

Results Cord Plasma brain derived neurotrophic factor level was significantly increased among cases compared to controls. Among cases, brain derived neurotrophic factor level at delivery and after $72 \mathrm{~h}$ significantly correlated with the severity of encephalopathy according to Sarnat staging being higher as severity increases. Brain derived neurotrophic factor level significantly increased after $72 \mathrm{~h}$ of life compared to its level at delivery among cases. Brain derived neurotrophic factor levels at delivery and at $72 \mathrm{~h}$ postnatal were predictors of severe Sarnat stage and poor outcome.

Conclusion We concluded that brain derived neurotrophic factor level as a marker of central nervous system insult is increased in full term newborns with perinatal asphyxia. It can serve as an indicator for the severity of encephalopathy and adverse outcomes.

\section{USE OF AMPLITUDE INTEGRATED ELECTROENCEPHALOGRAPHY IN NEWBORNS WITH SEVERE HYPERBILIRUBINEMIA}

doi:10.1136/archdischild-2012-302724.1095

M Chang, JH Shin. Department of Pediatrics, Chungnam National University Hospital, Daejeon, Republic of Korea

Background and Aims The spectrum of bilirubin-induced neurologic dysfunction (BIND) is very wide and the symptoms and signs may be very mild or absent. Amplitude-integrated electroencephalography (aEEG) allows continuous trend recording of cerebral function in high-risk newborns. However, published knowledge regarding correlation between aEEG and BIND remains limited.

In this study, we hypothesized that abnormal aEEG in infants with severe hyperbilirubinemia is useful for detection of asymptomatic BIND.

Methods This is a prospective observational study of newborns with severe hyperbilirubinemia in our NICU from April 2011 to December 2011. Patients were included if they were $\geq 34$ weeks gestational age (GA) at birth and their total serum bilirubin [TSB] $>20 \mathrm{mg} / \mathrm{dL}$. The aEEG was performed for 6 hours since admission, and rechecked when TSB is below $10 \mathrm{mg} / \mathrm{dL}$.

Results Fourteen infants were enrolled. Male to female was 10 to 4 . Their GA was $37.2 \pm 1.2$ weeks and their birth weight was $3,238 \pm 421 \mathrm{~g}$. Their peak TSB was $23.63 \pm 2.7 \mathrm{mg} / \mathrm{dL}$. Photopherapies were performed in all infants and exchange transfusions were also performed in 2 infants. Six out of 14 infants (42.8\%) showed abnormal aEEG findings such as discontinuity, abnormal cycling, depressed lower border and abnormal bandwidth span when their TSB were markedly elevated. However none had any noticeable symptoms or signs of neurologic dysfunction. All abnormal aEEG findings were normalized after treatment.
Conclusions Abnormal aEEG finding in infants with severe hyperbilirubinemia is useful for detection of asymptomatic BIND and can be reversible with appropriate treatment.

\section{ENIGMA OF MANAGEMENT OF SEIZURES IN HYPOXIC ISCHEMIC ENCEPHALOPATHY (HIE) - WHEN TO STOP ANTICONVULSANTS?}

doi:10.1136/archdischild-2012-302724.1096

'S Nangia, 'A Saili, ${ }^{2}$ A Garg. 'Division of Neonatology, Department of Pediatrics, ${ }^{2}$ Department of Pediatrics, Lady Hardinge Medical College and Kalawati Saran Children's Hospital, New Delhi, India

Background Although there is some agreement regarding what and when to initiate as anticonvulsant medication for seizures in HIE, there is no consensus about when to stop medication.

Objective To assess the effect of early stoppage of anticonvulsant drugs in HIE on seizure recurrence and neurological outcome.

Design/methods This prospective study enrolled neonates with HIE with non-metabolic seizures from August 2007 to July 2010. A loading dose of $20 \mathrm{mg} / \mathrm{kg}$ of phenobarbitone was used for seizure control. Additional mini-boluses of $5 \mathrm{mg} / \mathrm{kg}$ till a cumulative dose of $40 \mathrm{mg} / \mathrm{kg}$ followed by phenytoin was used if required.

Results Out of 59 neonates, $85 \%$ had cord pH below 7.2 and $83 \%$ had $\mathrm{BE}$ of -12 or higher. At birth $89 \%$ required positive pressure ventilation and $40 \%$ needed mechanical ventilation during NICU stay. 26/59(44\%) had seizure onset before $6 \mathrm{hrs}$ and 23/59 (39\%) between $6-12 \mathrm{hrs}$. $64 \%$ had a single episode of seizure, $22 \%$ had $2-3$ episodes and 10\% had 4-6 episodes. Twelve babies expired and 47 were discharged without anticonvulsant. There was no recurrence of seizure in $44 / 47(94 \%)$. On follow up at 3 months $40 / 47(85 \%)$, at 6 months $30 / 35(86 \%)$, at 12 months $25 / 29(86 \%)$, at 24 months $23 / 26(89 \%)$ and at 30 months $13 / 16(81 \%)$ had normal neurological outcome.

Conclusions This pilot work suggests that potentially apoptotic anticonvulsant drugs can probably be stopped early as soon as seizures abate in HIE without increased risk of seizure recurrence or adverse neurological outcome.

\section{ACTIVATED PROTEIN C DECREASES ENDOTOXIN-INDUCED INFLAMMATORY RESPONSES IN INFANTS WITH NEONATAL ENCEPHALOPATHY}

doi:10.1136/archdischild-2012-302724.1097

1,2,3,4 HO Eliwan, 2,3FO O'Hare, ${ }^{2} \mathrm{D}$ Sweetman, ${ }^{3} \mathrm{~W}$ Watson, ${ }^{3} \mathrm{~A}$ O'Neill, ${ }^{1,4,5,6} \mathrm{EJ}$ Molloy. ${ }^{1}$ Neonatology, Our Lady's Children's Hospital, Crumlin; ${ }^{2}$ Neonatology, National Maternity Hospital, Holles Street; ${ }^{3}$ UCD School of Medicine and Medical Science, Conway Institute for Biomolecular and Biomedical Science, University College Dublin; ${ }^{4}$ Royal College of Surgeon; ${ }^{5}$ National Maternity Hospital, Holles Street; ${ }^{6}$ UCD School of Medicine and Medical Science, Conway Institute for Biomolecular and Biomedical Science, Univeristy College Dublin, Dublin, Ireland

Introduction Infection and inflammation can be antecedents of neonatal encephaloapthy (NE) and increase the risk of neurological sequelae. Activated protein C (APC) has anticoagulant and antiinflammatory effects and provides neuroprotection in ischemic brain and spinal cord injury.

Aims To examine neutrophil and monocyte responses to Lipopolysaccharide (LPS) in infants with NE $(n=22)$ and also the effect of APC compared with healthy adult controls $(n=15)$.

Methods Whole blood was incubated with LPS +/-APC and TLR4, $\mathrm{CD} 11 \mathrm{~b}$ expression, and reactive oxygen intermediate (ROI) release from neutrophils and monocytes was examined by flow cytometry.

Results Neutrophil and monocyte CD11b expression was significantly increased in response to LPS in adults controls $(p<0.001)$ and NE infants $(p<0.001)$. However infants with NE were LPS-hyporesponsive 